\title{
The impact of electronic health record (EHR) interoperability on immunization information system (IIS) data quality
}

\author{
Mary Woinarowicz ${ }^{1}$, MA, Molly Howell ${ }^{1}$, MPH
}

1. North Dakota Department of Health, Division of Disease Control

\begin{abstract}
Objectives: To evaluate the impact of electronic health record (EHR) interoperability on the quality of immunization data in the North Dakota Immunization Information System (NDIIS). Methods: NDIIS doses administered data was evaluated for completeness of the patient and dose-level core data elements for records that belong to interoperable and non-interoperable providers. Data was compared at three months prior to electronic health record (EHR) interoperability enhancement to data at three, six, nine and twelve months post-enhancement following the interoperability go live date. Doses administered per month and by age group, timeliness of vaccine entry and the number of duplicate clients added to the NDIIS was also compared, in addition to, immunization rates for children $19-35$ months of age and adolescents $11-18$ years of age. Results: Doses administered by both interoperable and non-interoperable providers remained fairly consistent from pre-enhancement through twelve months post-enhancement. Comparing immunization rates for infants and adolescents, interoperable providers had higher rates both pre- and post-enhancement than non-interoperable providers for all vaccines and vaccine series assessed. The overall percentage of doses entered into the NDIIS within one month of administration varied slightly between interoperable and non-interoperable providers; however, there were significant changes between the percentage of doses entered within one day and within one week with the percentage entered within one day increasing and within one week decreasing with interoperability. The number of duplicate client records created by interoperable providers increased from 94 duplicates pre-enhancement to 10,552 at twelve months postenhancement, while the duplicates from non-interoperable providers only increased from 300 to 637 over the same period. Of the 40 core data elements in the NDIIS, there was some difference in completeness between the interoperable versus non-interoperable providers. Only middle name, sex, county, phone number, mother's maiden name, vaccine manufacturer, lot number and expiration date were significantly (>=5\%) different between the two provider groups. Conclusions: Interoperability with provider EHRs has had an impact on NDIIS data quality. Timeliness of data entry has improved and overall doses administered have remained fairly consistent, as have the immunization rates for the providers assessed. There are more technical and non-technical interventions that will need to be accomplished by NDIIS staff and vendor to help reduce the negative impact of duplicate record creation, as well as, data completeness.
\end{abstract}

Key Words: Immunization information systems, electronic health records, meaningful use, HL7, data quality

Correspondence: mary.woinarowicz@nd.gov

DOI: $10.5210 /$ ojphi.v8i2.6380 
This is an Open Access article. Authors own copyright of their articles appearing in the Online Journal of Public Health Informatics. Readers may copy articles without permission of the copyright owner(s), as long as the author and OJPHI are acknowledged in the copy and the copy is used for educational, not-for-profit purposes.

\section{Introduction}

Immunization Information Systems (IIS) are confidential, population based systems that record immunization administration data from participating providers, provide consolidated immunization histories at the point of care and provide aggregate data on vaccinations for use in surveillance to increase immunization rates and reduce vaccine-preventable disease [1]. In 1995, the Centers for Disease Control and Prevention (CDC) compiled a list of required and optional core data elements for IIS. The National Vaccine Advisory Committee (NVAC) reviewed and updated the IIS core data elements as part of their Initiative on Immunization Registries [2]. The CDC incorporated the NVAC recommendations and again updated the IIS core data elements in 2012 to correspond with the IIS functional standards and IIS Strategic Plan for 2013-2017 [3,4]. The purpose of the core data elements is to help standardize the capture of data in the IIS and to facilitate the consistent exchange of data between the IIS, electronic health record (EHR) systems and other IIS [2,3].

When the American Recovery and Reinvestment Act (ARRA) was enacted in 2009, one of its goals was to promote the adoption and increase the "meaningful use" of EHRs [5]. The Centers for Medicare and Medicaid Services (CMS), in coordination with the Office of the National Coordinator for Health Information Technology (ONC), have set different criteria that must be met by participating providers in order to receive an incentive payment. One of the public health reporting criteria included in all three stages of meaningful use is the electronic exchange of data between an EHR and an IIS [6]. This electronic exchange of data is referred to as interoperability. According to the Healthcare Information and Management Systems Society (HIMSS), "interoperability is the ability of different information technology systems...to...exchange data, and use the information that has been exchanged." [7]

In 2010, the North Dakota Department of Health (NDDoH) received ARRA funding to establish real-time, bidirectional interoperability connections between the North Dakota Immunization Information System (NDIIS) and the EHRs of the state's providers responsible for the highest volume of childhood (< 6 years) immunizations. Prior to 2010, the NDIIS was not interoperable with any providers. All providers entered immunizations directly into the NDIIS, in addition to entry into their EHR. As of August 31, 2013, the NDIIS was interoperable with 186 individual provider practices that represented more than half of all doses administered to children 18 and younger in the NDIIS.

The quality of data in an IIS is vital to its ability to determine a patient's immunization status, calculate immunizations due, assess immunization coverage or generate reminder and recall notices [6]. Without timely and accurate data entered into the NDIIS, it cannot support its basic 
functionality or provide a benefit to its users or the people in its jurisdiction [6]. The NDIIS has historically maintained high data quality and provider and patient participation by having a number of data quality checks in place that help ensure a high level of data quality. However, these checks are only visible to providers manually entering data directly into the NDIIS. Providers submitting data electronically (interoperable) do not see these same validation checks or warnings if data is not complete in their EHRs.

Other IIS have evaluated the completeness of immunization records in their IIS by comparing the IIS immunization records to the records in provider offices and other facilities in order to evaluate the impact of EHR interoperability. They have found that their IIS records have had more complete immunization information since it is a consolidated record and not just record of immunizations administered at one provider practice [8,9]. North Dakota requires reporting of childhood immunizations to the IIS. According to the 2013 IIS Annual Report, the NDIIS has $96.8 \%$ of North Dakota children $<6$ years of age, $83.4 \%$ of adolescents $11-17$ years of age and $77.9 \%$ of adults 19 years of age and older participating in the NDIIS [10]. Since the NDIIS already has excellent immunization record completeness, the NDDoH wanted to examine other areas of data quality related to the immunization record.

The objective of this study is to evaluate the impact of EHR interoperability on NDIIS data quality by comparing data entry, timeliness and data completeness, as well as, immunization rates for records belonging to providers submitting electronic data (i.e. interoperable) to those for providers manually entering data directly into the NDIIS (i.e. non-interoperable).

\section{Methods}

In the NDIIS, a “provider” refers to a clinic or immunization practice, not to a specific individual practitioner. A client record in the NDIIS is assigned to a provider practice if they were the last provider to enter a non-influenza vaccine in that client's record. Data for all clients in the NDIIS with doses administered between August 1, 2011 and August 31, 2014 was extracted from the NDIIS for evaluation. Extracted data elements included client first, middle and last name, birthdate, sex, birth state/country, race, ethnicity, address, phone number, mother's first, middle and last name, vaccine type, vaccination date (dose date), lot number and vaccine-level VFC eligibility IIS core data elements.

All data extracted was separated into one of two groups based on assigned provider; records belonging to interoperable providers (IPs) and non-interoperable providers (NIPs). An IP is defined as a provider whose EHR submits data to the NDIIS electronically (i.e. without human intervention) using messaging standards set by Health Level 7 (HL7) International and as defined by the HL7 implementation guide for immunization messaging [11,12]. A NIP is defined as a provider who manually enters data directly into the NDIIS user interface in addition to documenting in their EHR or paper records. They do not submit data to the NDIIS electronically. Pre-enhancement (before interoperability) data included doses administered between August 1 and October 31, 2011, prior to the first health system EHR connection to the NDIIS. Post-enhancement (after interoperability) data included doses administered between September 1, 2013 and August 31, 2014, three through twelve months after the final provider EHRs were connected to the NDIIS. 
The overall data entry analysis evaluated the total number of doses administered by IPs and NIPs per month and by age group for clients five years of age and younger, six to ten years, 11-18 years, 19-59 years and 60 years and older. Doses for each month were summed for three months preenhancement and three, six, nine and twelve months post-enhancement. The data entry analysis also evaluated the number of duplicate client records added by IPs and NIPs at three months preenhancement and three, six, nine and twelve months post-enhancement, as well as, the timeliness of all doses entered into the NDIIS less than one day after the dose administration date, within one week (i.e. one to seven days) of administration and within one month (i.e. 30 days) for the three months pre-enhancement and three, six, nine and twelve months post-enhancement.

Completeness of IIS core data elements for client and dose records that belong to IPs and were entered into the NDIIS three months pre-enhancement and at three, six, nine and twelve months post-enhancement were calculated and compared to the completeness of records for NIPs.

Immunization rates for children 19 - 35 months of age for the 4:3:1:3:3:1:4 (4 diphtheria, tetanus and acellular pertussis (DTaP), 3 polio, 1 measles, mumps and rubella (MMR), 3 hepatitis B, 3 haemophilus influenzae type B (Hib), 1 varicella and 4 pneumococcal conjugate (PCV)) vaccine series and for adolescents $11-18$ years of age for one dose of tetanus, diphtheria and acellular pertussis (Tdap) and meningococcal conjugate (MCV4) vaccines, two doses of varicella and three doses of human papillomavirus (HPV) vaccines were calculated and compared for clients that belong to IPs versus NIPs for three months pre-enhancement and three, six, nine and twelve months post-enhancement. SAS ${ }^{\circledR}$ 9.3, (SAS Institute, Inc., Cary, North Carolina) and Microsoft ${ }^{\circledR}$ Excel $^{\circledR} 2010$ (Microsoft Corp., Redmond, Washington) were used analyze all NDIIS data.

\section{Results}

Doses administered to the $6-10$ year, $11-18$ year, $19-59$ year and 60 years and older age groups by both IPs and NIPs remained fairly consistent from pre-enhancement to three, six, nine and twelve months post-enhancement. NIPs had a higher percentage of doses administered for all age groups with the exception of the $0-5$ year age group. Pre-enhancement NIPs entered $53 \%$ of the doses in the NDIIS compared to $47 \%$ for IPs. Post-enhancement, IPs entered $53 \%$ of doses at three months, $55 \%$ at six months, $56 \%$ at nine and twelve months (Figure 1 ).

Looking at the timeliness of data entry, the overall percentage of doses entered into the NDIIS within one month of administration varied slightly (less than $2 \%$ ) over the three months preenhancement and $4.6 \%$ over the twelve months post-enhancement. There were, however, some significant changes between the percentage of doses entered within one day and within one week. The percentage of doses entered within one day increased from $54.6 \%$ at the start of the preenhancement period to $79.5 \%$ at the end of the twelve months post-enhancement, while the doses entered within one week of administration decreased from $38.6 \%$ to $14.9 \%$ over the same time period (Figure 2). 


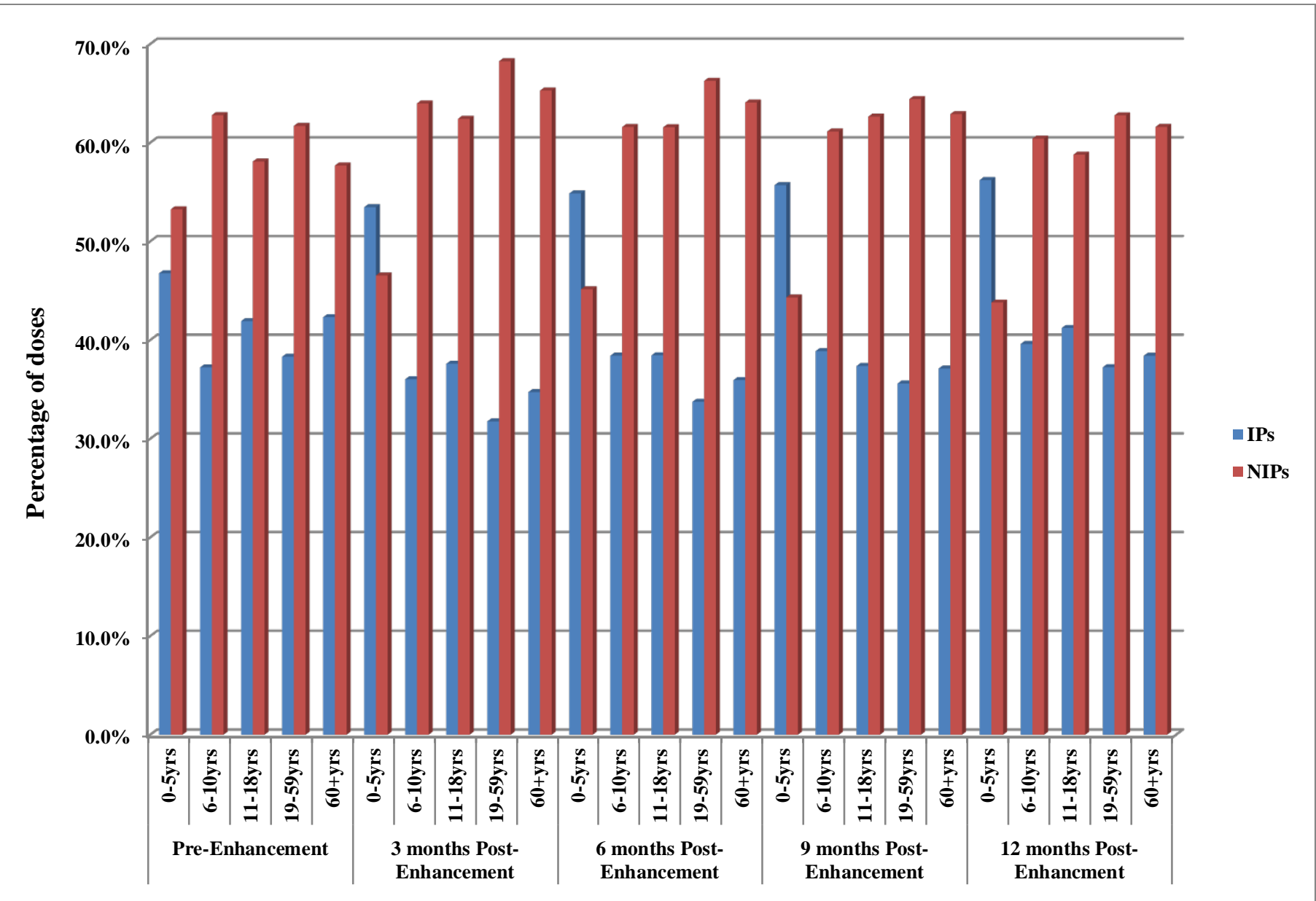

Figure 1. Comparison of percentage of doses administered to clients in the NDIIS by interoperable and non-interoperable providers

Data obtained from the NDIIS for doses added between August 1, 2011 and August 31, 2014.

Pre-enhancement, the number of duplicate client records created in the NDIIS by NIPs was more than three times the number created by IPs (300 vs. 94). Post-enhancement, the number of duplicate client records created by NIPs increased from 377 at three months to 637 at twelve months, whereas the number of duplicate client records created by IPs increased to 1,695 at three months post enhancement and continued to increase with 9,883 duplicates created at nine months and 10,552 at twelve months (Figure 3). 


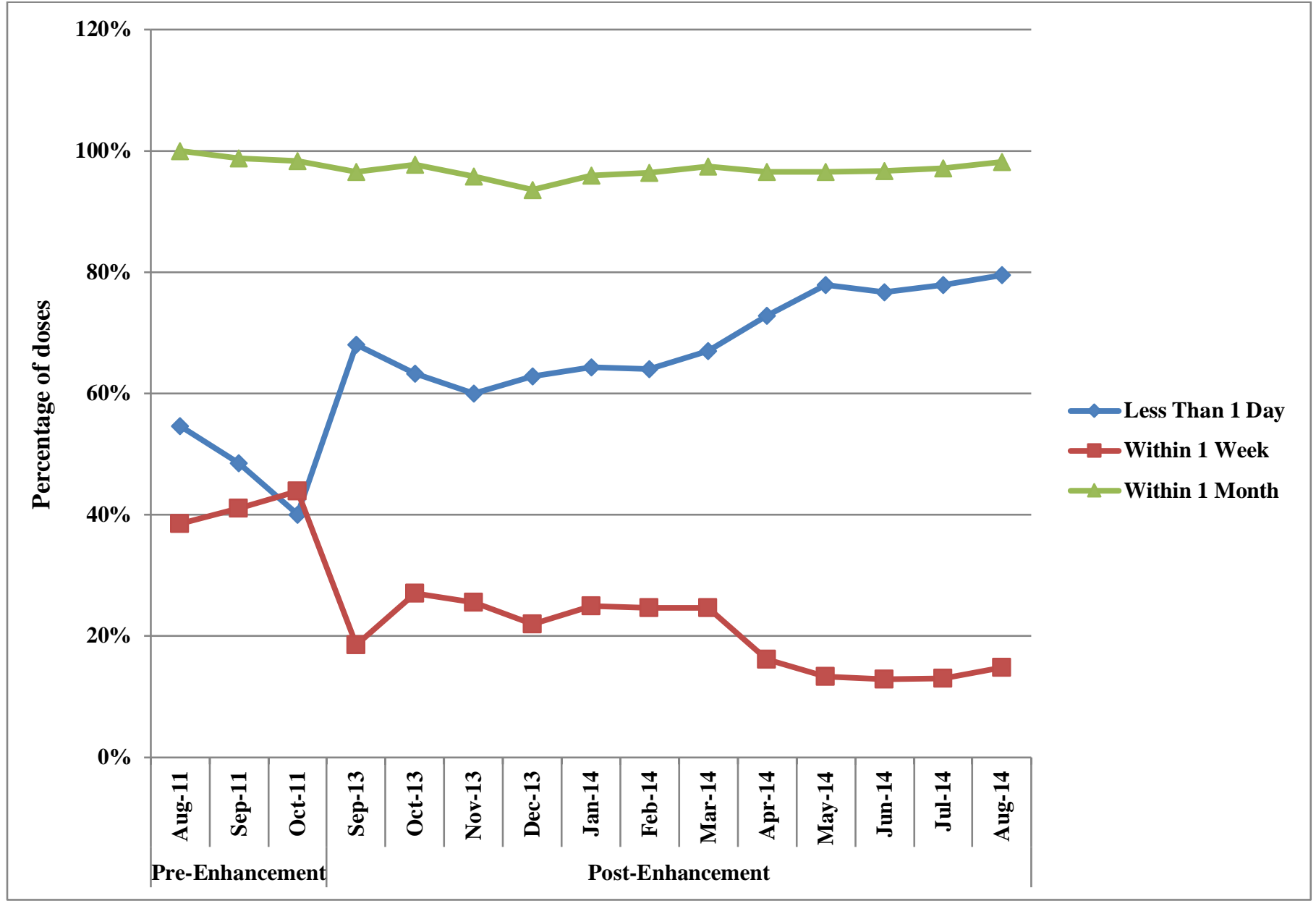

Figure 2. Overall timeliness of dose data entry by all providers in the NDIIS

Data obtained from the NDIIS for dose records added each month between August 1, 2011 and August 31, 2014.

Of the 40 core data elements in the NDIIS, completeness for only three elements, middle name, county and phone number, were significantly $(>=5 \%)$ higher for NIPs compared to IPs preenhancement. Completeness for only two data elements, sex and mother's maiden name, were significantly higher for IPs pre-enhancement. For all other data elements there was no significant difference in completeness between the interoperable versus NIPs. By twelve months postenhancement, there were five data elements: middle name, phone number, vaccine manufacturer, lot number and expiration date, significantly higher for NIPs and completeness for mother's maiden name was significantly higher for IPs. Additionally, completeness for sex showed almost no difference between IPs and NIPs post-enhancement and the percentage difference in completeness for county was only $2 \%$ post-enhancement versus $5 \%$ pre-enhancement (Table 1 ). 


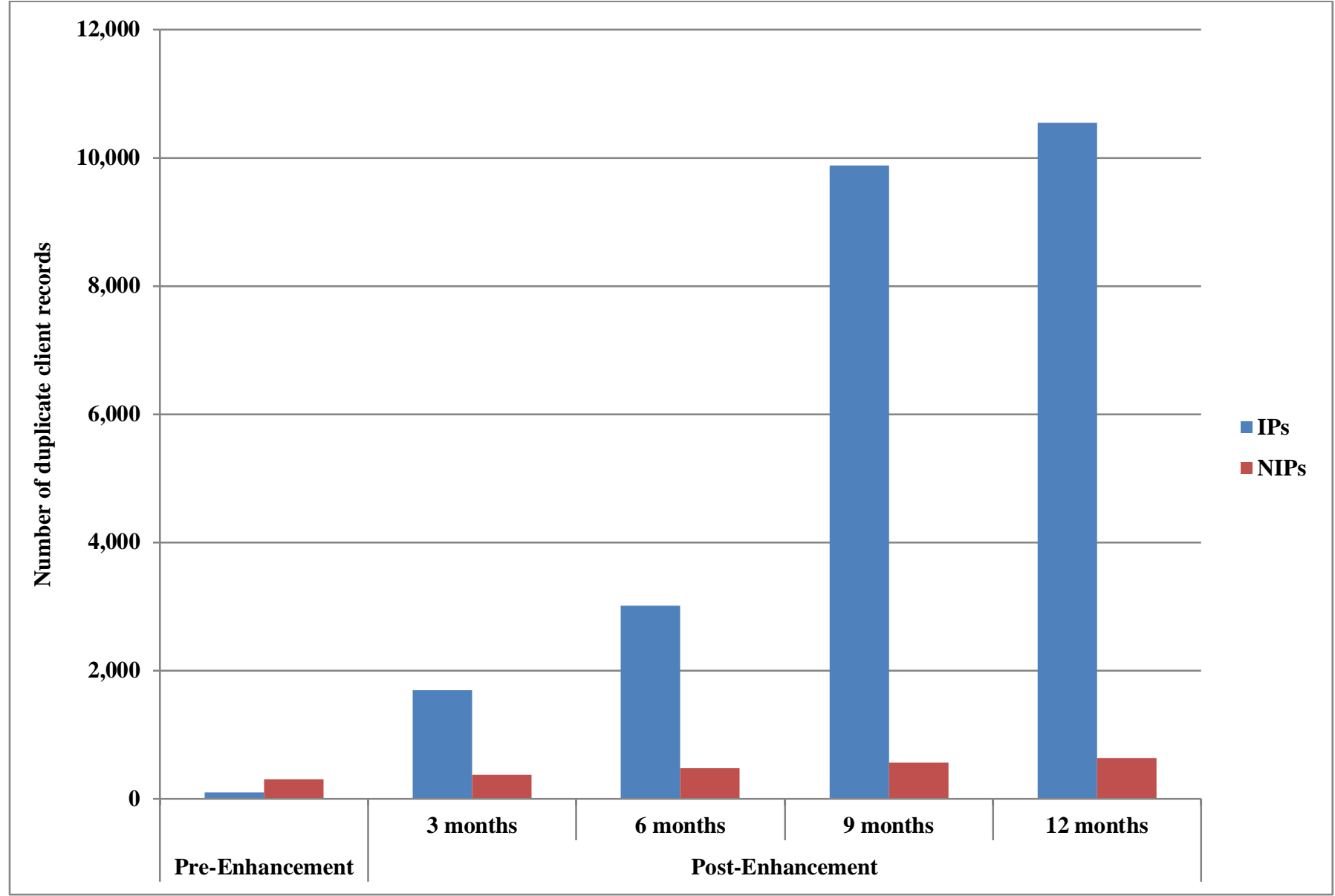

Figure 3. Comparison of the number of duplicate client records added to the NDIIS by interoperable and non-interoperable providers

Data obtained from the NDIIS for duplicate client records added to the NDIIS between August 1, 2011 and August 31, 2014.

Comparing immunization rates for infants and adolescents, IPs had higher rates both pre- and postenhancement than NIPs for all vaccines and vaccine series assessed. The rate for the infant 4:3:1:3:3:1:4 series was $82 \%$ for IPs pre-enhancement and $77 \%$ for NIPs, for a difference of only $5 \%$ between the two groups. By twelve months post-enhancement, there was a difference of 12\% with the IPs still having a higher percentage of their infants up-to-date with the complete series (70\%) when compared to the infants of NIPs (58\%) (Table 2). 
Table 1. Comparison of the Completeness of IIS Core Data Elements in the NDIIS for Interoperable and Non-Interoperable Providers

Data obtained from the NDIIS for records added between August 1, 2011 and August 31, 2014.

\begin{tabular}{|l|l|l|l|l|l|l|}
\hline $\begin{array}{l}\text { IIS Core Data } \\
\text { Element }\end{array}$ & $\begin{array}{l}\text { Pre- } \\
\text { Enhanceme } \\
\text { nt IPs }\end{array}$ & $\begin{array}{l}\text { Pre- } \\
\text { Enhanceme } \\
\text { nt NIPs }\end{array}$ & $\begin{array}{l}\text { Differen } \\
\text { ce }\end{array}$ & $\begin{array}{l}\text { Post- } \\
\text { Enhanceme } \\
\text { nt IPs }\end{array}$ & $\begin{array}{l}\text { Post- } \\
\text { Enhanceme } \\
\text { nt NIPs }\end{array}$ & $\begin{array}{l}\text { Differen } \\
\text { ce }\end{array}$ \\
\hline MIDDLE NAME & $72.4 \%$ & $84.1 \%$ & $-11.7 \%$ & $64.1 \%$ & $80.3 \%$ & $-16.2 \%$ \\
\hline SEX & $99.9 \%$ & $94.9 \%$ & $5.0 \%$ & $96.9 \%$ & $96.8 \%$ & $0.1 \%$ \\
\hline $\begin{array}{l}\text { MOTHER'S } \\
\text { MAIDEN NAME }\end{array}$ & $46.8 \%$ & $40.0 \%$ & $6.8 \%$ & $55.8 \%$ & $44.1 \%$ & $11.7 \%$ \\
\hline $\begin{array}{l}\text { COUNTY } \\
\text { NUONEER }\end{array}$ & $93.8 \%$ & $98.4 \%$ & $-4.6 \%$ & $96.4 \%$ & $98.7 \%$ & $-2.3 \%$ \\
\hline $\begin{array}{l}\text { VACCINE } \\
\text { MANUFACTURE }\end{array}$ & $71.0 \%$ & $70.4 \%$ & $0.6 \%$ & $68.0 \%$ & $84.4 \%$ & $-16.4 \%$ \\
\hline ROT NUMBER & $70.1 \%$ & $69.0 \%$ & $1.1 \%$ & $64.7 \%$ & $83.0 \%$ & $-18.3 \%$ \\
\hline $\begin{array}{l}\text { EXPIRATION } \\
\text { DATE }\end{array}$ & $70.1 \%$ & $69.0 \%$ & $1.1 \%$ & $64.7 \%$ & $83.0 \%$ & $-18.3 \%$ \\
\hline
\end{tabular}

The differences in the up-to-date rates for adolescent clients at IPs versus NIPs varied by vaccine and time period. For one dose of Tdap, the biggest difference was at twelve months post enhancement with a rate for IPs of $79 \%$ compared to $66 \%$ for NIPs; for one dose of MCV4 and two doses of varicella, the biggest differences were seen pre-enhancement with a MCV4 rate of $81 \%$ and a varicella rate of $50 \%$ for IPs compared to only $66 \%$ and $35 \%$ for NIPs. Rates for HPV and two doses of MCV4 had the smallest differences between interoperable and NIPs with variations of $5 \%$ or less at each time interval (Table 3 ). 
Table 2. Comparison of the Percentage of North Dakota Infants (19 - 35 months) at Interoperable and Non-Interoperable Providers Up-To-Date with Recommended Immunizations (4:3:1:3:3:1:4 series)

Data obtained from the NDIIS for client records as of October 31, 2011, November 30, 2013, February 28, 2014, May 31, 2014 and August 31, 2014.

\begin{tabular}{|l|l|l|l|l|}
\hline $\begin{array}{l}\text { Interoperability } \\
\text { Status }\end{array}$ & $\begin{array}{l}\text { Time } \\
\text { Period }\end{array}$ & IPs & NIPs & $\begin{array}{l}\text { Differenc } \\
\text { e }\end{array}$ \\
\hline \multirow{5}{*}{ Pre-Enhancement } & $\begin{array}{l}\mathbf{3} \\
\text { month } \\
\text { s }\end{array}$ & $82 \%$ & $77 \%$ & $5 \%$ \\
\hline \multirow{5}{*}{ Post-Enhancement } & $\begin{array}{l}\mathbf{3} \\
\text { month } \\
\text { s }\end{array}$ & $78 \%$ & $72 \%$ & $6 \%$ \\
\hline & $\begin{array}{l}\mathbf{6} \\
\text { month } \\
\text { s }\end{array}$ & $72 \%$ & $66 \%$ & $6 \%$ \\
\cline { 2 - 6 } & $\begin{array}{l}\mathbf{9} \\
\text { month } \\
\text { s }\end{array}$ & $72 \%$ & $59 \%$ & $12 \%$ \\
\hline & $\begin{array}{l}\mathbf{1 2} \\
\text { month } \\
\text { s }\end{array}$ & $70 \%$ & $58 \%$ & $13 \%$ \\
\hline
\end{tabular}

Table 3. Comparison of the Percentage of North Dakota Adolescents at Interoperable and Non-Interoperable Providers Up-To-Date with Recommended Immunizations

Data obtained from the NDIIS for client records in the NDIIS as of October 31, 2011, November 30, 2013, February 28, 2014, May 31, 2014 and August 31, 2014.

\begin{tabular}{|l|l|l|l|l|}
\hline $\begin{array}{l}\text { Interoperability Status and } \\
\text { Vaccine }\end{array}$ & $\begin{array}{l}\text { Time } \\
\text { Period }\end{array}$ & IPs & NIPs & Difference \\
\hline Pre-Enhancement Tdap & $\mathbf{3}$ months & $78 \%$ & $64 \%$ & $13 \%$ \\
\hline \multirow{3}{*}{ Post-Enhancement Tdap } & $\mathbf{3}$ months & $82 \%$ & $74 \%$ & $8 \%$ \\
\cline { 2 - 5 } & $\mathbf{6}$ months & $71 \%$ & $63 \%$ & $8 \%$ \\
\cline { 2 - 5 } & $\mathbf{9}$ months & $73 \%$ & $62 \%$ & $11 \%$ \\
\cline { 2 - 5 } & $\begin{array}{l}\mathbf{1 2} \\
\text { months }\end{array}$ & $79 \%$ & $66 \%$ & $13 \%$ \\
\hline
\end{tabular}


The impact of electronic health record (EHR) interoperability on immunization information system (IIS) data quality

\begin{tabular}{|c|c|c|c|c|}
\hline Pre-Enhancement 1 MCV4 & 3 months & $81 \%$ & $66 \%$ & $15 \%$ \\
\hline \multirow{4}{*}{ Post-Enhancement 1 MCV4 } & 3 months & $83 \%$ & $75 \%$ & $9 \%$ \\
\hline & 6 months & $66 \%$ & $57 \%$ & $8 \%$ \\
\hline & 9 months & $68 \%$ & $57 \%$ & $11 \%$ \\
\hline & $\begin{array}{l}2 \\
\text { months }\end{array}$ & $76 \%$ & $64 \%$ & $13 \%$ \\
\hline Pre-Enhancement 2 MCV4 & 3 months & $9 \%$ & $8 \%$ & $1 \%$ \\
\hline \multirow{4}{*}{ Post-Enhancement 2 MCV4 } & 3 months & $32 \%$ & $27 \%$ & $5 \%$ \\
\hline & 6 months & $14 \%$ & $13 \%$ & $1 \%$ \\
\hline & 9 months & $18 \%$ & $15 \%$ & $3 \%$ \\
\hline & \begin{tabular}{|l|}
12 \\
months
\end{tabular} & $29 \%$ & $22 \%$ & $7 \%$ \\
\hline Pre-Enhancement Varicella & 3 months & $50 \%$ & $35 \%$ & $15 \%$ \\
\hline \multirow{4}{*}{ Post-Enhancement Varicella } & 3 months & $65 \%$ & $58 \%$ & $7 \%$ \\
\hline & 6 months & $71 \%$ & $64 \%$ & $7 \%$ \\
\hline & 9 months & $72 \%$ & $61 \%$ & $11 \%$ \\
\hline & \begin{tabular}{|l|}
12 \\
months
\end{tabular} & $72 \%$ & $61 \%$ & $11 \%$ \\
\hline Pre-Enhancement HPV & 3 months & $15 \%$ & $11 \%$ & $4 \%$ \\
\hline \multirow{4}{*}{ Post-Enhancement HPV } & 3 months & $23 \%$ & $18 \%$ & $4 \%$ \\
\hline & 6 months & $20 \%$ & $17 \%$ & $3 \%$ \\
\hline & 9 months & $22 \%$ & $18 \%$ & $4 \%$ \\
\hline & $\begin{array}{l}12 \\
\text { months }\end{array}$ & $25 \%$ & $20 \%$ & $5 \%$ \\
\hline
\end{tabular}

\section{Discussion}

Based on the results of the doses administered analysis pre and post enhancement, EHR interoperability did not have a significant impact, with very little change in the percentage of doses 
administered between IPs and NIPs for all but one of the age groups assessed. There was a difference with the percentage of doses administered to clients in the $0-5$ year age group which we would expect to see since the IPs administer the highest volume of immunizations to children under six. The overall changes in the percentage of doses administered, however, were not significant.

Interoperability has had a positive impact on timeliness of doses entered into the NDIIS. Although the percentage of doses coming into the NDIIS within one month has remained fairly consistent, the NDIIS is receiving more data entered the same day that the dose was administered, with fewer doses taking one week or more. Improved timeliness of entry into the NDIIS makes it easier for providers and other NDIIS users to make more informed decisions about a patient's immunization status, current and future immunization needs and to conduct timelier reminder/recall.

A significant impact of NDIIS interoperability is the increase in the number of duplicate client records being created. The number of duplicate client records being added to the NDIIS has increased considerably after EHR interoperability. When electronic messages are sent from an EHR to the NDIIS, the client information is matched based on an exact match of first name, last name and birthdate. If an exact match cannot be found, a new record is created in the NDIIS. There are a lot of different naming conventions in EHR systems with some systems allowing special characters but not spaces, others only allowing spaces, or users entering patients in an EHR with a nickname instead of their full given name. All of the EHR differences can vary from what the NDIIS will allow for names, causing records not to match. Additionally, the process for identifying and removing duplicate client records from the NDIIS is a manual and time consuming process which involves the manual review of the two or more duplicate records, merging the demographic and immunization data into one complete record and updating the status of the duplicate records to "deleted." Records are not completely removed from the NDIIS so that data can be restored if an error occurred and records should not have been merged.

In April 2014, the NDIIS implemented an automated vaccine de-duplication system that evaluates all incoming dose records for potential duplicate records based on guidance from the American Immunization Registry Association (AIRA) Data Quality Assurance in Immunization Information Systems: Incoming Data Modeling of Immunization Registry Operations Work Group (MIROW) guide [13]. Since being implemented, this system has removed more than 400,000 duplicate dose records. A similar, automated process is needed to help more easily identify and remove duplicate client records. Additionally, improvements to the algorithm used when looking for matching records are needed to better match clients from the electronic HL7 message to the NDIIS, so that fewer duplicate records are created in the first place. These improvements include searching for a match based on additional data elements, such as middle name and sex and to adjust for potential differences in naming conventions by removing spaces and special characters from the client's name before searching for a match.

Overall, completeness of core data elements is one of the biggest data quality challenges. Both the sending EHR and the NDIIS have to follow the published HL7 guidelines for the electronic transmission of immunization data, but there are some differences between the release of the implementation guide (IG) supported and a data element required in the NDIIS [12]. Additionally, 
data being sent from an EHR can still be considered compliant with the HL7 IG, but does not provide quality data to the NDIIS. For example, the NDIIS requires a client middle name; an EHR may not capture this data element or may automatically send a value of "NA." They are still sending the data element in the HL7 message and in a valid format, but the information is not meaningful in the NDIIS. This is also the case with data fields like race and ethnicity and having an "unknown" value sent from an EHR. In early 2014, the NDIIS vendor implemented changes to the NDIIS interoperability messaging system that would evaluate the source of client demographic information and only make updates where necessary instead of automatically overriding the current NDIIS data with data from the EHR. For example, if a client record is already in the NDIIS with a valid value for race and the EHR sends an "unknown" value the NDIIS data will not be changed to "unknown" as a result. More updates could be done to this algorithm in order to ensure the best data is being kept or added to the NDIIS.

Data completeness for vaccine lot number, manufacturer and expiration date is tied together. The NDDoH immunization program requires that providers enrolled in the federal Vaccines for Children (VFC) Program use the NDIIS to manage their public vaccine inventory, but they do not have to do the same for their privately purchased vaccine [14]. For providers entering directly into the NDIIS, the lot number field in the NDIIS dose entry workflow is not a free-text field; it is a drop-down selection tied to a provider's vaccine inventory. If a provider does not have a specific lot number in their NDIIS inventory, the lot number won't be available for selection and cannot be entered into an individual dose record. Because the manufacturer and expiration date are tied to the lot number in the provider's inventory, if a lot number is not documented, neither are the other two data elements. This also means that when a lot number is electronically sent from an EHR to the NDIIS in an HL7 message, there must be an exact matching lot number in the provider's inventory. If an exact match is not found in the NDIIS, the lot number and its corresponding data will not be added to the dose record and a dummy dose will be added instead. In the NDIIS, a dummy dose is when the vaccine abbreviation is entered in place of the administered lot number when the lot number is not known. This could lead to a high percentage of dose records without a lot number, manufacturer or expiration date, even if the information was sent to the NDIIS. Without accurate lot numbers documented, doses can't be decremented from a provider's inventory and in the case of a vaccine recall or storage and handling incident; only doses with an accurate lot number could be identified using the NDIIS. This would cause individuals who should be notified of the recall and possibly need to be revaccinated to be missed. Updates to the matching algorithm that looks for a lot number match between the HL7 message from the EHR and the NDIIS provider inventory are needed to try and account for common data entry mistakes, such as replacing a letter "B” with a number "8” or a letter “O” with a number “0.”

\section{Conclusion}

Interoperability with provider EHRs has had both positive and negative impacts on NDIIS data quality. Timeliness of data entry has improved and overall doses administered have remained fairly consistent, as have the immunization rates for the providers assessed. There is additional work that will need to be done by NDIIS staff and its vendor to help reduce the negative impact of duplicate record creation, as well as, data completeness. 
Moving forward, continuous monitoring of data completeness and overall data quality will continue to be a challenge, but vital in order to ensure that the information being sent from the EHR is complete and that it is being added to the NDIIS correctly. Additional analysis is needed to understand why the data is not being sent to the NDIIS, if the data being sent is incorrect or if the data is not being added to the NDIIS correctly. Once the issues have been identified, data corrections will need to be made in order to maintain the best possible data quality in the NDIIS. As more and more providers submit immunization data electronically, new challenges will arise, making the need for improvements and changes now that much more important.

Despite the challenges related to data quality and the need for continual data quality monitoring post-interoperability, the benefits of increased timeliness of data entry and the overall increase in data being entered into the IIS can be seen based on the results of this study. Increased data and improved timeliness will enable better use of the IIS for determining a patient's immunization status, calculating immunizations due, assessing immunization coverage and generating reminder and recall notices as well as for delivering consolidated immunization records and supporting their immunization program efforts to reduce vaccine preventable disease [1,6].

\section{Limitations}

All of the data for this analysis was extracted from the NDIIS retrospectively. This means that even though the date ranges used to query data from the NDIIS were in keeping with pre and postenhancement time intervals, the data may have been changed since it was originally entered. Corrections may have been made to the data that would have improved the data quality even though it was originally incorrect. Additionally, any data in records currently marked as "deceased" in the NDIIS were excluded from all of the analyses even though the client may not have been deceased at the pre or post-enhancement interval.

The NDIIS client status for duplicate client records is simply "deleted.” This status is seldom used for any other reason than to remove a duplicate record; however it is possible that a client record has been marked as "deleted" but they were not actually a duplicate record. Records for deceased clients may have duplicates that were also marked as "deceased" and would not have been counted as a duplicate record. Both of these limitations on client status could have a minor impact on the analysis of duplicate records.

\section{Conflicts of Interest}

The authors have no financial relationships relevant to this article.

\section{Disclosure}

The findings and conclusions in this report are those of the authors and do not necessarily represent the official position of the NDDoH or the Centers for Disease Control and Prevention. This project was supported by the Health Resources and Services Administration (HRSA) of the U.S. Department of Health and Human Services (HHS) under grant number 5H23IP000784-02 and title 
for grant amount of $\$ 183,440.00$. This information or content and conclusions are those of the author inferred by HRSA, HHS or the U.S. Government.

\section{References}

1. Immunization Information System (IIS). (2012, May 15) About Immunization Information Systems: What is IIS? Retrieved November 23, 2015 from CDC website: http://www.cdc.gov/vaccines/programs/iis/about.html

2. Immunization Information Systems NVAC Progress Report February 2007

3. Immunization Information System (IIS). (2012, May 15) IIS Recommended Core Data Elements. Retrieved November 23, 2015 from CDC website: http://www.cdc.gov/vaccines/programs/iis/core-data-elements.html

4. Immunization Information System (IIS). (2012, May 15) IIS Functional Standards. Retrieved November 23, 2015 from CDC website: http://www.cdc.gov/vaccines/programs/iis/funcstds.html

5. Electronic Health Records (EHR) Incentive Programs. (2015, October 29) About the EHR Incentive Program. Retrieved November 23, 2015 from CMS website: https://www.cms.gov/Regulations-and-

Guidance/Legislation/EHRIncentivePrograms/index.html?redirect=/EHRIncentivePrograms/

6. Immunization Information System (IIS). (2012, May 15) Meaningful Use and Immunization Information Systems. Retrieved November 23, 2015 from CDC website: http://www.cdc.gov/vaccines/programs/iis/meaningful-use/index.html

7. What is Interoperability? (2013, April 5) HIMSS, Transforming Health through Information Technology. Retreived June 13, 2016 from HIMSS website: http://www.himss.org/library/interoperability-standards/what-is-interoperability

8. Kolasa MS, Cherry JE, Chilkatowsky AP, Reyes DP, Lutz JP. 2005. Practice-based electronic billing systems and their impact on immunization registries. J Public Health Manag Pract. 11(6), 493-99. http://dx.doi.org/10.1097/00124784-200511000-00004

9. Koepke R, Petit AB, Ayele RA, Jens CE, Schauer SL, et al. 2015. Completeness and Accuracy of the Wisconsin Immunization Registry: An Evaluation Coinciding with the Beginning of Meaningful Use. $J$ Public Health Manag Pract. 21(3), 273-81. http://dx.doi.org/10.1097/PHH.0000000000000216

10. Immunization Information System (IIS). (2014, September 9) IIS Annual Report (IISAR): 2013 IISAR Data Participation Rates Retrieved December 22, 2015 from CDC website: http://www.cdc.gov/vaccines/programs/iis/annual-report-iisar/2013-data.html\#adult 
11. Health Level Seven INTERNATIONAL. (@2007-2016) Introduction to HL7 Standards Retrieved April 27, 2016 from http://www.hl7.org/implement/standards/index.cfm?ref=nav

12. Immunization Information System (IIS). (2012, August 1) IIS Health Level 7 (HL7) Implementation Guidance. Retrieved November 23, 2015 from CDC website: http://www.cdc.gov/vaccines/programs/iis/technical-guidance/hl7.html

13. American Immunization Registry Association. (2008). Data Quality Assurance in Immunization Information Systems: Incoming Data. Retrieved from: http://www.immregistries.org/AIRA_MIROW_Chap3_DQA_02112008.pdf

14. Vaccines for Children Program (VFC). (2014, February 14) The VFC Program: At a Glance. Retrieved from http://www.cdc.gov/vaccines/programs/vfc/about/index.html 